\title{
ESCRT-III-mediated membrane repair in cell death and tumor resistance
}

\author{
Jiao Liu $^{1} \cdot$ Rui Kang ${ }^{2} \cdot$ Daolin Tang $\mathbb{B}^{2}$ \\ Received: 15 May 2020 / Revised: 23 June 2020 / Accepted: 7 July 2020 / Published online: 15 July 2020 \\ (c) The Author(s), under exclusive licence to Springer Nature America, Inc. 2020
}

\begin{abstract}
The plasma membrane is made of glycerophospholipids that separate the inner and outer parts of the cell. Under physiological conditions, it acts as a barrier and gatekeeper to protect cells from the environment. In pathological situations, it undergoes structural and functional changes, resulting in cell damage. Indeed, plasma membrane damage caused by various stresses (e.g., hypoxia, nutritional deficiencies, ultraviolet radiation, and chemotherapeutic agents) is one of the hallmarks of cell death. Phosphatidylserine exposure and plasma membrane blebbing usually occurs in apoptotic cells, while necrotic cells lose the integrity of the plasma membrane and thereby release intracellular damage-associated molecular patterns. In contrast, the endosomal sorting complex required for transport-III (ESCRT-III), an evolutionarily conserved protein complex with membrane fission machinery, plays a key role in the repair of damaged plasma membranes in various types of regulated cell death, such as necroptosis, pyroptosis, and ferroptosis. These emerging findings indicate that ESCRTIII is a potential target to overcome drug resistance during tumor therapy.
\end{abstract}

Cancer remains a huge challenge for human health worldwide. Although early chemotherapy is used to quickly relieve tumors, tumor cells may develop drug resistance after treatment, resulting in disease recurrence [1]. There may be two main ways to solve the problem of single-drug resistance. One is combined drug therapy, which has been developed into increasingly complex treatment plans for patients. The other is the use of different dose strengths, including strong short-interval chemotherapy or high-dose chemotherapy. Nevertheless, the efficacy of chemotherapy has been stagnant for many years. In contrast, the development of new and different therapies, such as targeted therapies for tyrosine kinases [2], as well as immunotherapy using immune-checkpoint inhibitors [3], has been an important leap in recent years. Either way, the ultimate goal is to kill tumor cells and protect normal cells. Therefore, it is

Daolin Tang

daolin.tang@utsouthwestern.edu

1 The Third Affiliated Hospital, Guangzhou Medical University, Guangzhou, Guangdong 510510, China

2 Department of Surgery, UT Southwestern Medical Center, Dallas, TX 75390, USA important to understand the signals and mechanisms of cell death and survival during tumor therapy.

According to the recommendations of the Nomenclature Committee on Cell Death, cell death can be divided into accidental cell death (ACD) and regulated cell death (RCD) [4]. In short, ACD is a passive and uncontrolled process, while RCD is an active and controlled process [4]. RCD can be further classified into apoptotic and nonapoptotic cell death that shows different morphological, genetic, and biochemical characteristics [5]. Since resistance to apoptosis is one of the main obstacles to the successful treatment of cancer, there has been a great interest in the development of reagents to trigger various forms of nonapoptotic cell death (e.g., necroptosis [6, 7], pyroptosis [8, 9], ferroptosis $[10,11]$, alkaliptosis $[12,13]$, and parthanatos $[14,15])$ to suppress tumor growth. However, similar to the results previously observed in conventional chemotherapy to induce apoptosis, tumor cells still have resistance to targeted nonapoptotic cell death. Thus, tumor cells may develop a common drug-resistance mechanism to avoid cell death, resulting in treatment failure.

The integrity of the plasma membrane is critical to cell homeostasis [16]. It is composed of a phospholipid bilayer with a hydrophobic interior and a hydrophilic exterior that undergo continuous remodeling. Phosphatidylcholine and sphingomyelin are mainly present on the outer leaflet, 


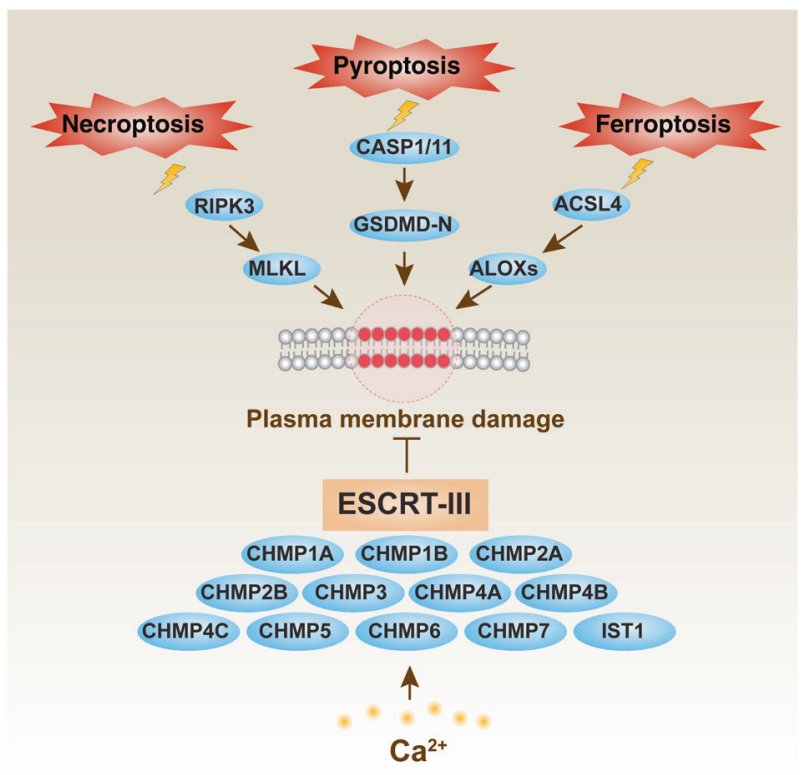

Fig. 1 ESCRT-dependent membrane repair negatively regulates cell death. Necroptosis is a caspase-independent cell death mediated by receptor-interacting protein kinase 3 (RIPK3)-dependent MLKL activation. GSDMD mediates pyroptosis after its cleavage by caspase 1 or caspase 11 to produce an $\mathrm{N}$-terminal fragment, namely GSDMD$\mathrm{N}$. Ferroptosis is a lipid peroxidation-dependent cell death that is mediated by the activation of acyl-coA synthetase long-chain family member 4 (ACSL4) and lipoxygenases (ALOXs). All these cell death stimuli can cause plasma membrane damage through different mechanisms. In contrast, $\mathrm{Ca}^{2+}$ influx-mediated activation and recruitment of ESCRT-III complex leads to the repair of damaged plasma membranes during cell death.

whereas phosphatidylethanolamine and phosphatidylserine mainly accumulate on the inner leaflet. Molecules and gases with low hydrophobicity (e.g., oxygen and carbon dioxide) will quickly pass through the plasma membrane. In contrast, the membrane limits the diffusion of highly charged molecules (e.g., ions) and large molecules (e.g., sugars and amino acids). Changes in the plasma membrane during cell death depend on the context. The exposure of phosphatidylserine to the outer plasma membrane is not only an early event of apoptosis, but also a signal of "eat me" to promote its clearance by myeloid cells [17]. Unlike the shrinking and blebbing of plasma membranes in apoptotic cells, an increase in plasma membrane permeability and finally membrane rupture occur in necrotic cells [18]. The destruction of the physical barrier of the plasma membrane can lead to cell death and subsequent inflammation responses through the release of cellular contents, especially damage-associated molecular patterns [19].

The last 10 years have witnessed tremendous progress in research on membrane repair [20, 21]. In particular, membrane repair mediated by the endosomal sorting complexes required for transport (ESCRT) plays a key role in the response to various mechanical and chemical stresses [22].
ESCRT is assembled into machinery with multiple subunits on the cytosol, and it drives membrane scission or sealing involved in multiple physiological and pathological processes (e.g., microvesicle formation, autophagosome formation, plasma membrane repair, cytokinetic abscission, formation of viral replication compartments, lysosome repair, nuclear pore-quality control, and nuclear envelope repair) [22]. It is an evolutionarily conserved mechanism, originally part of a so-called vacuolar protein-sorting mutant found in yeast [23]. Among its five functionally distinct subcomplexes (ESCRT-0, ESCRT-I, ESCRT-II, ESCRT-III, and VPS4), the ESCRT-III complex plays a unique role in suppressing various types of RCD, such as necroptosis [24], pyroptosis [25], and ferroptosis [26], by repairing damaged plasma membranes (Fig. 1).

In mammalian cells, the ESCRT-III complex consists of 12 subunits: charged multivesicular body protein 1A (CHMP1A), CHMP1B, CHMP2A, CHMP2B, CHMP3, CHMP4A, CHMP4B, CHMP4C, CHMP5, CHMP6, CHMP7, and increased sodium tolerance 1 (IST1) [27]. The destruction of the integrity of the plasma membrane leads to a large influx of $\mathrm{Ca}^{2+}$, thereby stimulating the rapid recruitment of ESCRT-III components to damaged plasma membranes during cell death. In addition to the $\mathrm{Ca}^{2+}$ signal, the recruitment of ESCRT-III is further modulated by several endogenous proteins, including annexin A7 (ANXA7), ALG2 alpha-1,3/1,6-mannosyltransferase (ALG2), programmed cell death 6-interacting protein (PDCD6IP, also known as ALIX), and tumor susceptibility 101 (TSG101), that control membrane budding and scission [28-30]. Ultimately, the activation of ESCRT-III machinery leads to membrane repair by shedding damaged parts of cell membranes. Thus, high levels of components of ESCRT-III contribute to resistance to cell death. In contrast, the inhibition of ESCRT-III machinery through genetic depletion of its core components increases susceptibility to anticancer agent-induced cell death (Fig. 1). For example, gene deletion of CHMP2A or CHMP4B promotes mixed-lineage kinase domain-like pseudokinase (MLKL)-dependent necroptosis [24], whereas the silencing of CHMP3 enhances the N-terminal fragment of gasdermin D (GSDMD-N)mediated pyroptosis [25]. Further, gene deletion of CHMP5 or CHMP6 increases the anticancer activity of ferroptosis activators (e.g., erastin and RSL3) [26]. Genetic variation of ESCRT-III components, such as CHMP4C, is also associated with increased susceptibility to cancer tumorigenesis [31, 32], further supporting the pathological role of a deregulated ESCRT-III pathway in human cancers.

In summary, there is increasing evidence showing that ESCRT-III subunits assemble into spiral filaments that mediate membrane remodeling and scission during anticancer agent-induced cell death. These results are inspiring interest in ESCRT-III as a target in order to 
modulate the expression of this pathway for therapeutic applications. However, the precise mechanisms and effects of different components of ESCRT-III machinery on different types of RCD are still poorly understood. It is also difficult to distinguish membrane repair-dependent and -independent functions of ESCRT-III during cell death. In recent years, studies using cryogenic electron tomography have identified multiple membraneinteraction surfaces for the helical assembly of ESCRT polymers $[33,34]$. The subcomplex specificity may be determined by residues in the stalk, which is important for cargo sorting [23, 35]. Further structural studies of the ESCRT-III subunits may facilitate the development of specific small-molecule compounds to target membranerepair pathways in cancer therapy. Genotype specificity and tumor microenvironment may ultimately affect the efficacy of this anticancer strategy. Considering that this membrane-repair mechanism also exists in normal cells, the short- and long-term side effects of ESCRT-IIItargeted therapy in cancer treatment need further evaluation.

Acknowledgements We thank Dave Primm (Department of Surgery, University of Texas Southwestern Medical Center) for his critical reading of the paper. D.T. was supported by grants from the US National Institutes of Health (R01CA229275) and the American Cancer Society (Research Scholar Grant RSG-16-014-01-CDD [D.T.]).

\section{Compliance with ethical standards}

Conflict of interest The authors declare that they have no conflict of interest.

Publisher's note Springer Nature remains neutral with regard to jurisdictional claims in published maps and institutional affiliations.

\section{References}

1. Nikolaou M, Pavlopoulou A, Georgakilas AG, Kyrodimos E. The challenge of drug resistance in cancer treatment: a current overview. Clin Exp Metastasis. 2018;35:309-18.

2. Pottier C, Fresnais M, Gilon M, Jerusalem G, Longuespee R, Sounni NE. Tyrosine kinase inhibitors in cancer: breakthrough and challenges of targeted therapy. Cancers. 2020;12:731-48.

3. Ribas A, Wolchok JD. Cancer immunotherapy using checkpoint blockade. Science. 2018;359:1350-5.

4. Galluzzi L, Vitale I, Aaronson SA, Abrams JM, Adam D, Agostinis P, et al. Molecular mechanisms of cell death: recommendations of the Nomenclature Committee on Cell Death 2018. Cell Death Differ. 2018;25:486-541.

5. Tang D, Kang R, Berghe TV, Vandenabeele P, Kroemer G. The molecular machinery of regulated cell death. Cell Res. 2019;29:347-64.

6. Qin X, Ma D, Tan YX, Wang HY, Cai Z. The role of necroptosis in cancer: a double-edged sword? Biochim Biophys Acta Rev Cancer. 2019;1871:259-66.
7. Gong Y, Fan Z, Luo G, Yang C, Huang Q, Fan K, et al. The role of necroptosis in cancer biology and therapy. Mol Cancer. 2019;18:100

8. Fang Y, Tian S, Pan Y, Li W, Wang Q, Tang Y, et al. Pyroptosis: a new frontier in cancer. Biomed Pharmacother. 2020;121:109595.

9. Xia X, Wang X, Cheng Z, Qin W, Lei L, Jiang J, et al. The role of pyroptosis in cancer: pro-cancer or pro-"host"? Cell Death Dis. 2019;10:650.

10. Xie Y, Hou W, Song X, Yu Y, Huang J, Sun X, et al. Ferroptosis: process and function. Cell Death Differ. 2016;23:369-79.

11. Stockwell BR, Friedmann Angeli JP, Bayir H, Bush AI, Conrad M, Dixon SJ, et al. Ferroptosis: a regulated cell death nexus linking metabolism, redox biology, and disease. Cell. 2017; 171:273-85.

12. Liu J, Kuang F, Kang R, Tang D. Alkaliptosis: a new weapon for cancer therapy. Cancer Gene Ther. 2020;27:267-9.

13. Song X, Zhu S, Xie Y, Liu J, Sun L, Zeng D, et al. JTC801 induces $\mathrm{pH}$-dependent death specifically in cancer cells and slows growth of tumors in mice. Gastroenterology. 2018;154: 1480-93.

14. David KK, Andrabi SA, Dawson TM, Dawson VL. Parthanatos, a messenger of death. Front Biosci (Landmark Ed). 2009;14:1116-28.

15. Susin SA, Lorenzo HK, Zamzami N, Marzo I, Snow BE, Brothers GM, et al. Molecular characterization of mitochondrial apoptosisinducing factor. Nature. 1999;397:441-6.

16. Harayama T, Riezman $H$. Understanding the diversity of membrane lipid composition. Nat Rev Mol Cell Biol. 2018;19:281-96.

17. Elmore S. Apoptosis: a review of programmed cell death. Toxicol Pathol. 2007;35:495-516.

18. Vanden Berghe T, Linkermann A, Jouan-Lanhouet S, Walczak H, Vandenabeele P. Regulated necrosis: the expanding network of non-apoptotic cell death pathways. Nat Rev Mol Cell Biol. 2014;15:135-47.

19. Tang D, Kang R, Coyne CB, Zeh HJ, Lotze MT. PAMPs and DAMPs: signal 0s that spur autophagy and immunity. Immunol Rev. 2012;249:158-75.

20. Horn A, Jaiswal JK. Cellular mechanisms and signals that coordinate plasma membrane repair. Cell Mol Life Sci. 2018;75: 3751-70.

21. Andrews NW, Corrotte M. Plasma membrane repair. Curr Biol. 2018;28:R392-7.

22. Vietri M, Radulovic M, Stenmark H. The many functions of ESCRTs. Nat Rev Mol Cell Biol. 2020;21:25-42.

23. Schmidt O, Teis D. The ESCRT machinery. Curr Biol. 2012;22: R116-20.

24. Gong YN, Guy C, Olauson H, Becker JU, Yang M, Fitzgerald P, et al. ESCRT-III Acts Downstream of MLKL to Regulate Necroptotic Cell Death and Its Consequences. Cell. 2017;169: 286-300. e16.

25. Ruhl S, Shkarina K, Demarco B, Heilig R, Santos JC, Broz P. ESCRT-dependent membrane repair negatively regulates pyroptosis downstream of GSDMD activation. Science. 2018;362:956-60.

26. Dai E, Meng L, Kang R, Wang X, Tang D. ESCRT-III-dependent membrane repair blocks ferroptosis. Biochem Biophys Res Commun. 2020;522:415-21.

27. McCullough J, Frost A, Sundquist WI. Structures, functions, and dynamics of ESCRT-III/Vps4 membrane remodeling and fission complexes. Annu Rev Cell Dev Biol. 2018;34:85-109.

28. Sonder SL, Boye TL, Tolle R, Dengjel J, Maeda K, Jaattela M, et al. Annexin A7 is required for ESCRT III-mediated plasma membrane repair. Sci Rep. 2019;9:6726.

29. Morita E, Sandrin V, Chung HY, Morham SG, Gygi SP, Rodesch $\mathrm{CK}$, et al. Human ESCRT and ALIX proteins interact with proteins of the midbody and function in cytokinesis. EMBO J. 2007;26:4215-27. 
30. Scheffer LL, Sreetama SC, Sharma N, Medikayala S, Brown KJ, Defour A, et al. Mechanism of $\mathrm{Ca}(2)(+)$-triggered ESCRT assembly and regulation of cell membrane repair. Nat Commun. 2014;5:5646.

31. Sadler JBA, Wenzel DM, Williams LK, Guindo-Martinez M, Alam SL, Mercader JM, et al. A cancer-associated polymorphism in ESCRT-III disrupts the abscission checkpoint and promotes genome instability. Proc Natl Acad Sci USA. 2018;115:E8900-8.

32. Mattissek C, Teis D. The role of the endosomal sorting complexes required for transport (ESCRT) in tumorigenesis. Mol Membr Biol. 2014;31:111-9.
33. Moser von Filseck J, Barberi L, Talledge N, Johnson IE, Frost A, Lenz M, et al. Anisotropic ESCRT-III architecture governs helical membrane tube formation. Nat Commun. 2020;11:1516.

34. Bertin A, de Franceschi N, de la Mora E, Maiti S, Alqabandi M, Miguet N, et al. Human ESCRT-III polymers assemble on positively curved membranes and induce helical membrane tube formation. Nat Commun. 2020;11:2663.

35. Flower TG, Takahashi Y, Hudait A, Rose K, Tjahjono N, Pak AJ, et al. A helical assembly of human ESCRT-I scaffolds reversetopology membrane scission. Nat Struct Mol Biol. 2020;27: $570-80$. 